\title{
A Low-Noise Double-Dipole Antenna SIS Mixer at 1 THz
}

\author{
S. V. Shitov \\ Institute of Radio Engineering and Electronics (IREE), Russian Academy of Sciences \\ 101999 Moscow, Russia \\ B. D. Jackson, A. M. Baryshev \\ Space Research Organization of the Netherlands (SRON) \\ P.O.Box 800, 9700 AV Groningen, the Netherlands \\ N. N. Iosad, J.-R. Gao and T. M. Klapwijk \\ Department of Applied Physics, Delft University of Technology (DIMES) \\ 2628 CJ, Delft, the Netherlands
}

\begin{abstract}
A quasi-optical mixer employing a $\mathrm{Nb} / \mathrm{Al} / \mathrm{AlO}_{\mathrm{x}} / \mathrm{Nb}$ twin-SIS junction with a $\mathrm{NbTiN} / \mathrm{SiO}_{2} / \mathrm{Al}$ microstrip coupling circuit is tested at 800-1000 GHz. The mixer design is developed as an option for HIFI frequency bands 3 and 4. The double-dipole antenna is made from NbTiN/Al; a Nb film is used for the back reflector. The mixer design is optimized for the IF band of 4-8 GHz. The receiver noise temperature $T_{R X}=250 \mathrm{~K}$ (DSB) is measured at $935 \mathrm{GHz}$ for the bath temperature $2 \mathrm{~K}$ at $\mathrm{IF}=1.5 \mathrm{GHz} ; \mathrm{T}_{\mathrm{RX}}$ remains below $350 \mathrm{~K}$ within the frequency range $850-1000 \mathrm{GHz}$. The double-dipole antenna beam pattern demonstrated good symmetry with sidelobes below $-16 \mathrm{~dB}$.
\end{abstract}

\section{$\underline{\text { Introduction }}$}

Low-noise THz-band heterodyne receivers are needed to realize the full potential of airborne and space-based telescopes currently being developed for sub-millimeter spectral astronomy, e. g. for HIFI [1]. To consider the possibility of designing an effective SIS mixer at $1 \mathrm{THz}$, it is worth to have a brief look at the most critical parameters of SIS mixers.

The $\mathrm{Nb} / \mathrm{Al} / \mathrm{AlO}_{\mathrm{x}} / \mathrm{Nb}$ SIS mixers are known as the quantum (photon) noise limited heterodyne down-converters [2], which are tested within frequency range $30-1500 \mathrm{GHz}$ and shown to yield receiver noise temperatures as low as $(2-3)^{*} \mathrm{hf} / \mathrm{k}_{\mathrm{B}}$ below $680 \mathrm{GHz}$, the gap frequency of $\mathrm{Nb}$ [3], [4]. Theoretically, the frequency range of all-Nb SIS mixers as quantum limited detectors can be extended up to twice the gap frequency, i. e. up to about $1300 \mathrm{GHz}$ [5]. However, a single junction SIS mixer can not cover the entire band because of its high specific capacitance, C, yielding the Q-factor of the circuit of order of 10 at $1 \mathrm{THz}$. The high Q-factor leads to at least two distinctive problems: a relatively narrow instantaneous bandwidth and increased influence of loss. The real part of the high frequency impedance of a superconducting film [6] is responsible for losses, which are growing as the square of the rf current density in the tuning circuit, i. e. proportional to 
$\mathrm{Q}^{2}$. The Q-factor can, theoretically, be reduced with increasing the junction current density, $\mathrm{J}_{\mathrm{c}}$. However, it is hard to do practically due to degradation of the IV-curve at $\mathrm{J}_{\mathrm{c}}>10-15 \mathrm{kA}$ [7]. There is, however, a possibility to reduce the $r f$ losses employing smaller (sub-micron area) junctions. Nevertheless, it is clear that quantum-limited SIS mixers are hard to realize for a frequency range where losses in the tuning circuit are essential. Unfortunately, the $r f$ circuits from $\mathrm{Nb}$ become lossy above $680 \mathrm{GHz}$ that is twice lower than the possible operational range of a Nb-based SIS junction. That is why, along with the efforts on high current density and sub-micron patterning of SIS junctions, the development of low-loss tuning circuits, in both electrodynamics and materials [8][12], is of great importance for realization of a THz-band quantum-limited SIS mixer.

The capacitance of the $r f$ tuning circuit can contribute to the problem of matching the IF port of the mixer to a standard $50 \Omega$ output transmission line due to high dynamic resistance of the SIS junction (up to about $1 \mathrm{k} \Omega$ ). To reduce this effect, which is more pronounced at higher IF and can also lead to a high Q-value, the capacitance of all tuning and coupling circuits has to be minimized along with use of a special IF matching circuit.

Two main solutions are available for a $r f$ feed of a SIS mixer: the waveguide and the open structure. All problems mentioned above are common for both types of mixers. However, a few specific problems exist in production of precision mechanical components for waveguides: corrugated horn antenna, waveguide channel, backshort structure, chip channel, etc. Another critical steps in production of a waveguide mixer are precise dicing and polishing of the SIS chips along with their alignment in the mixer block. In contrast, the quasioptical mixers can be fabricated on relatively large and easyto-handle substrates, which are a part of the optical system and dependent mostly on accuracy of the photolithography. However, the antenna beam pattern of a lens-antenna is dependent on quality of the microwave lens and the alignment accuracy of the chip [13], [14]. The theoretical level of the first order sidelobes is about $-18 \mathrm{~dB}$ for most known integrated lens-antennas that is higher than for the corrugated horn antennas used for waveguide SIS mixers.

The double-slot and the double-dipole are two types of antennas widely used in SIS mixers [10], [15]-[18]. Both antennas have, theoretically, similar parameters of the beam if used as an integrated lens-antenna; they have also similar values of the feed-point impedance. In spite a bare slot-antenna operated near its second resonance has a bit higher sidelobes in the H-plane (5\% or $-13 \mathrm{~dB})$ [17], the beam of the lens-antenna can be somewhat corrected by the microwave lens [13], [14]. The double-dipole antenna is used successfully for the integrated receiver [16]. The advantage of a slot-antenna is the shielding ground plane, which allows placing relatively complex circuits very close to the antenna. Unlike the dipole antenna, it is not possible however to return the backlobe using a reflector for the slot antenna. The double-dipole antenna usually has no capacitive coupling elements, so the total capacitance of the structure can be minimized that is important at high IF.

The challenge of the project was to demonstrate a quasioptical double-dipole antenna SIS mixer complying with HIFI requirements for the bands 3 and 4 [1]. 


\section{General Approach}

We have chosen the twin-junction SIS mixer with the anti-phase feed circuit [10] integrated with the double-dipole antenna, as shown in Figure 1; the numerical and experimental studies [10], [18] have demonstrated wider instantaneous bandwidth along with lower loss in comparison with the end-loaded tuning structure. The lower loss can be attributed to the lower $r f$ current density at the input of the impedance transformer, that is a specific feature of the twin-SIS junction. In spite the all-NbTiN microstrip can, at least potentially, be a low-loss at $1 \mathrm{THz}$, the $\mathrm{NbTiN} / \mathrm{SiO}_{2} / \mathrm{Al}$ tuning circuit has been chosen for two reasons: (1) it minimizes effects of heat-trapping observed previously in $\mathrm{Nb}$-based SIS junctions integrated with an all-NbTiN tuning circuit [19]; (2) the poor nucleation of NbTiN on top of the $\mathrm{SiO}_{2}$ layer may reduce the effective gap frequency of a wiring layer [20]. Two identical mixers are placed at the distance of $500 \mu \mathrm{m}$ in the center of a high-resistivity silicon chip of size $2 \mathrm{~mm}$ × $2 \mathrm{~mm}$ x $300 \mu \mathrm{m}$. These dimensions allow to accept a non-truncated 120-degree beam of a printed antenna. The second device can be used as a replacement. The diameter of a silicon elliptical lens was chosen to be $10 \mathrm{~mm}$, that defines the alignment accuracy of the chip of about $10 \mu \mathrm{m}$. The IF coupling circuit has to operate at the dynamic resistance of the mixer $\mathrm{R}_{d}=100 \Omega(200 \Omega$ per junction). The reflection loss has to be kept below $-10 \mathrm{~dB}$ within the IF band of 4-8 $\mathrm{GHz}$ for $\mathrm{R}_{\mathrm{d}}=50-150 \Omega$.

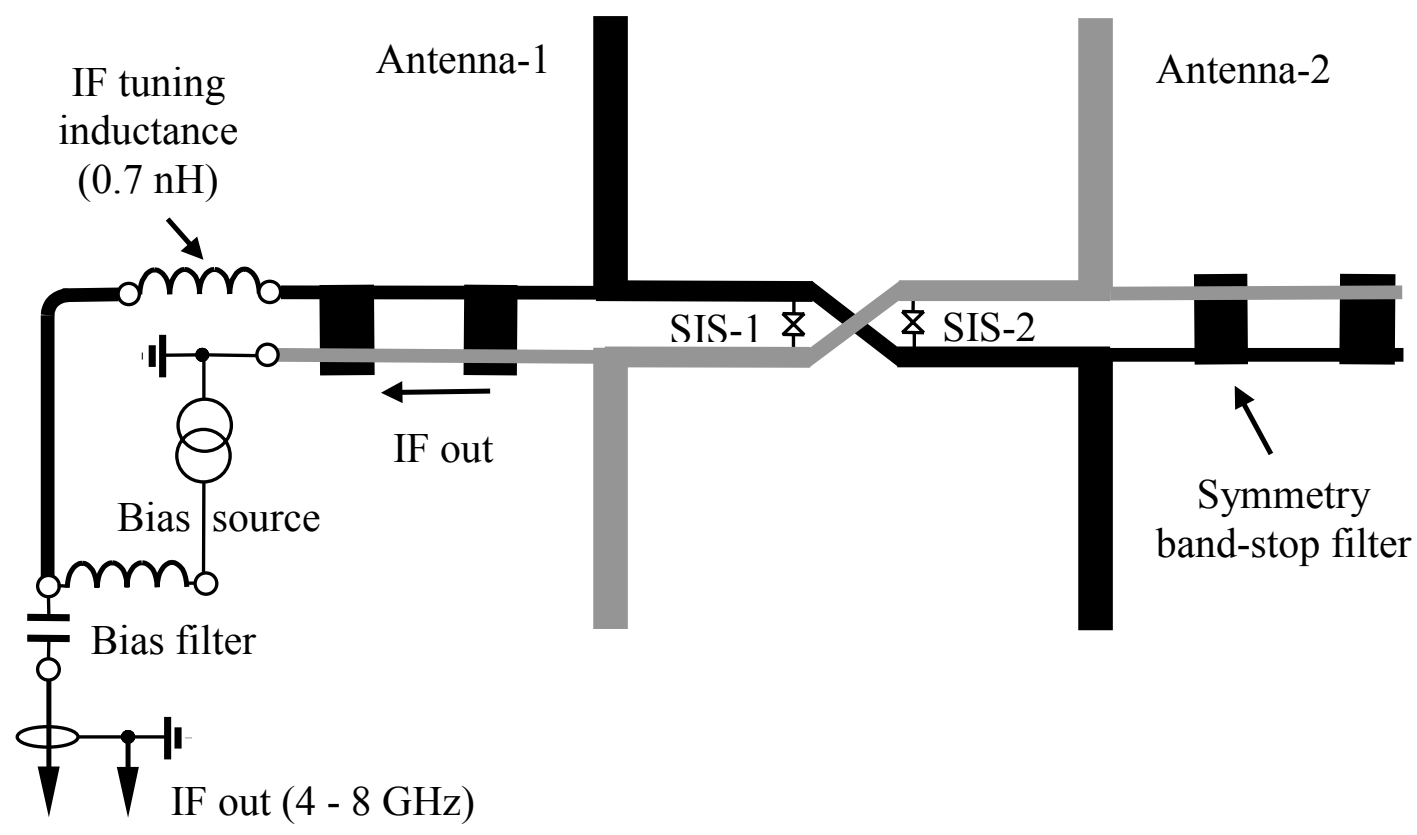

Fig. 1 Simplified electric scheme of quasi-optical planar SIS mixer with double-dipole antenna. The anti-phase mode of the twin-SIS junction (SIS-1 and SIS-2) is used in the mixer. Note that the junctions are connected in parallel for $d c$ current, but in series at $r f$. 


\section{Details of Design}

The devices are designed for two HIFI bands: $800-960 \mathrm{GHz}$ and $960-1120 \mathrm{GHz}$. The optimization of the layout is made for each of two bands separately. The double-dipole antenna from Figure 2 is scaled from $500 \mathrm{GHz}$ integrated receiver [16]. The size of the antenna is $34 \mu \mathrm{m} \times 40 \mu \mathrm{m}$ and the width of its arms is $4 \mu \mathrm{m}$. It was initially considered that almost any metal (e.g. $\mathrm{Nb}$ ) can be used for the back reflector at about $1 \mathrm{THz}$ assuming low current density at the surface of the film. The quarter-wavelength-thick silicon chip $(0.5 \mathrm{~mm} \times 0.5 \mathrm{~mm} \times 22 \mu \mathrm{m})$ one-side covered with $200 \mathrm{~nm}$ of $\mathrm{Nb}$ is placed onto the antenna as the back reflector. The calculated feed point impedance of the double-dipole antenna with the back reflector is shown in Figure 3. The anti-reflection coating of the microwave lens is optimized for $960 \mathrm{GHz}$ using the $46-\mu \mathrm{m}$ layer of Stycast $^{\mathrm{TM}} 1264$ epoxy $(\varepsilon \approx 2.9)$.

Two 1-micron area SIS junctions $\mathrm{Nb} / \mathrm{Al} / \mathrm{AlO}_{\mathrm{x}} / \mathrm{Nb}$ are integrated with the antenna as shown in Figure 1 and Figure 2. The transmission line connecting the two junctions (placed $3.6 \mu \mathrm{m}$ or $3 \mu \mathrm{m}$ apart) and antennas is a microstrip line with narrow ground plane - just $1 \mu \mathrm{m}$ wider than the strip which is $3 \mu \mathrm{m}$ or $4 \mu \mathrm{m}$ wide for the two bands respectively. Due to the nearly symmetric structure of the transmission line its strip and ground plane can be exchanged, so they are equally connected to the antennas picking up the anti-phase signals. The symmetry of the coupling structure provides also a virtual ground (a zero potential plane) at halfway between the junctions, that is equal to a tuning inductor connected in parallel to each junction at high frequency [10].

The calculated coupling at $r f$ is presented in Figure 4 along with the best fit of the experimental FTS data. In these calculations the base electrode from NbTiN is assumed to be a perfect superconductor with London penetration depth $300 \mathrm{~nm}$. The surface (sheet) resistance of $0.15 \Omega$ was used for Al. Two band-stop filters are connected to the double-dipole antenna, as shown in

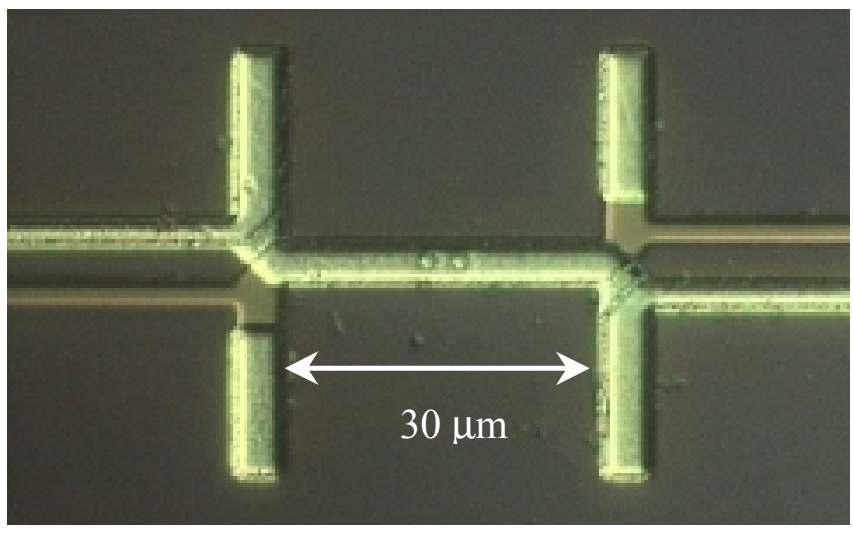

Fig. 2 Photograph of the double-dipole antenna SIS mixer fabricated on silicon substrate. The light metal strips are Al wiring; the darker ones are $\mathrm{NbTiN}$ strips at the bottom.
Figure 1, but only one filter is connected to the IF channel providing the best symmetry of the structure at $r f$. This is assumed to be essential for symmetry of the beam of the array antenna. To achieve a combination of compactness and efficiency, the filters are designed with alteration of coplanar strips and microstrips. It is found that losses in the antenna band-stop filters are not negligible. The 3\% absorption of the $r f$ signal is estimated for the three-stage bandstop filter. This value can be actually made lower by adding extra 

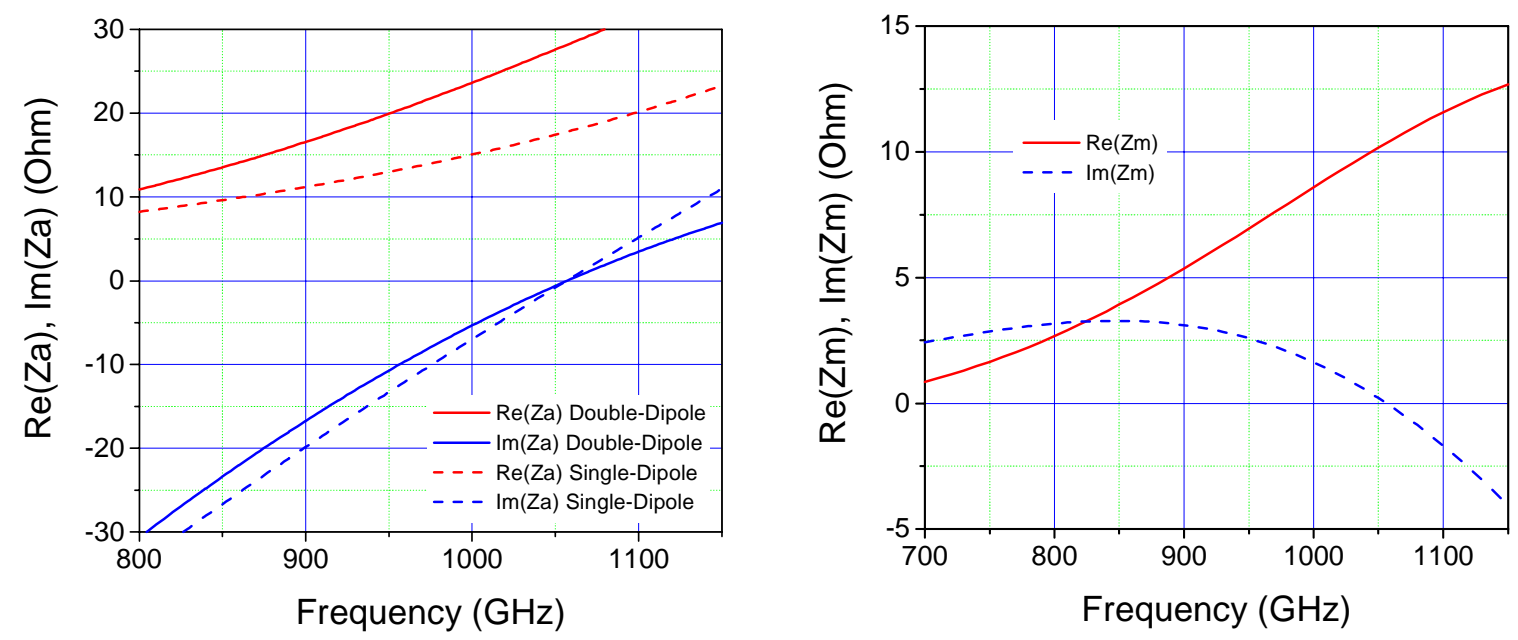

Fig. 3 Feed-point impedance calculated for the planar double-dipole antenna on semiinfinite silicon medium with a quarter-wave reflector (left graph); mutual impedance of the antenna array is plotted on the right graph.

sections, but the IF coupling would suffer from the growing capacitance of the filters. To obtain curves shown in Figure 4, both stray inductance of the SIS "tablet" and stray inductance of the microstrip, that is caused by the greater density of the $r f$ current near the junction window, are included in the calculation. The stray inductance can be estimated roughly as $0.1-0.2 \mathrm{pH}[21]$.

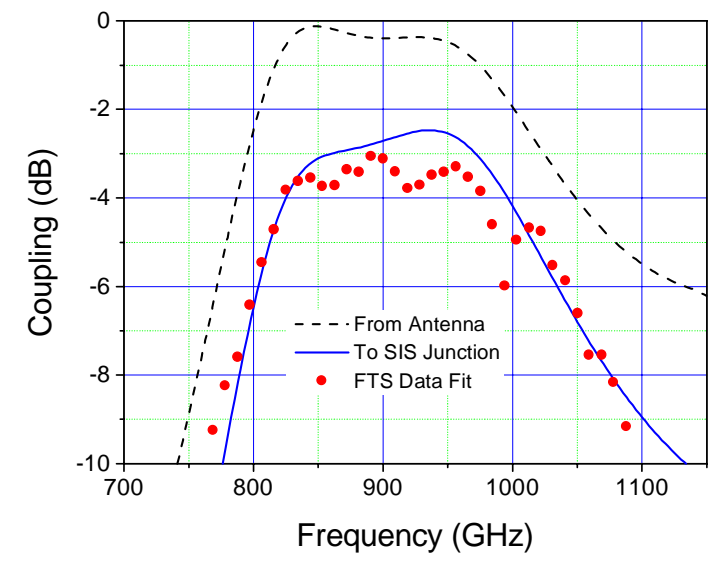

Fig. 4 Best fit of FTS data to the calculated coupling between the antenna and the mixing SIS junction. The drop of the antenna coupling (dashed) to the junction (solid) is caused by surface loss in the aluminum layer of the $\mathrm{NbTiN} / \mathrm{SiO}_{2} / \mathrm{Al}$ tuning stripline.

\section{Experimental Results}

The mixers are fabricated using a process similar to that described previously for waveguide devices with $\mathrm{NbTiN}$ and $\mathrm{Al}$ tuning circuits [20]. The standard optical lithography is used. The NbTiN ground plane $300 \mathrm{~nm}$ thick is deposited at ambient temperature. The $\mathrm{SiO}_{2}$ dielectric layer is $250 \mathrm{~nm}$ thick. The Al wiring layer $(400 \mathrm{~nm})$ has conductivity at $4 \mathrm{~K}$, $\sigma_{4 \mathrm{~K}} \approx 2 \times 10^{8} \Omega^{-1} \mathrm{~m}^{-1}$, and is expected to be in the anomalous limit [22]. The Al wiring layer is protected against chemical attack by $200 \mathrm{~nm}$ of $\mathrm{SiO}_{2}$. The typical IV-curve of the experimental twin-junction is presented in Figure 5. A few devices of band 4 (960$1120 \mathrm{GHz}$ ) were tested in the standard vacuum cryostat with optimized IR filters, 
which optical losses are estimated as $1.2 \mathrm{~dB}$ at $1 \mathrm{THz}$ [23]. The FTS data demonstrated a feature that looks very much like a high-frequency cutoff just above $1 \mathrm{THz}$. This feature was found to be almost independent on variation in the junction size and other important tuning parameters. The noise

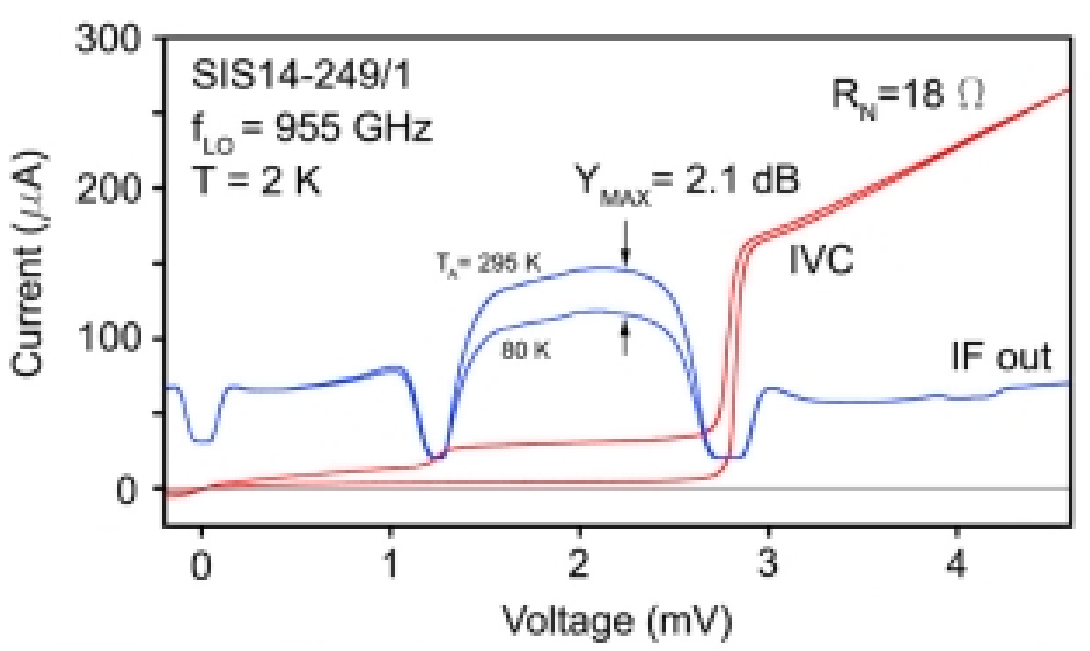

Fig. 5 Unpumped and pumped IV-curves of experimental SIS mixer along with hot/cold response at $955 \mathrm{GHz}$ measured at $\mathrm{IF}=1.5 \mathrm{GHz}$. Bath temperature is about $2 \mathrm{~K}$.

temperature of about

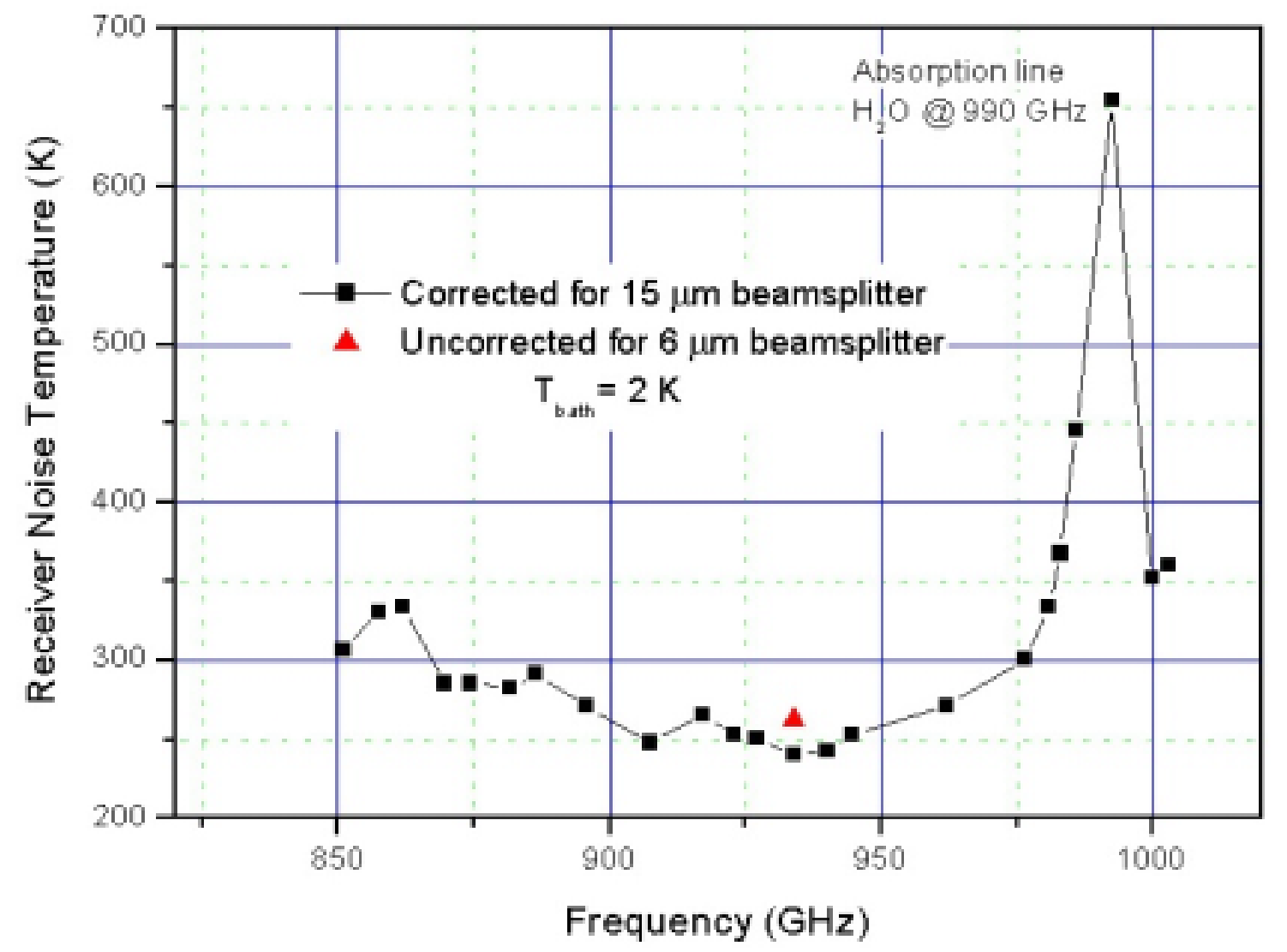

Fig. 6 Receiver noise temperature (DSB) measured for double-dipole antenna SIS mixer at bath temperature about $2 \mathrm{~K}$. 
$500 \mathrm{~K}$ was measured for a couple of devices above $950 \mathrm{GHz}$. A device with the tuning frequency a bit lower than $1 \mathrm{THz}$ was eventually selected. This device demonstrated a $3 \mathrm{~dB}$ FTS range of $800-1050 \mathrm{GHz}$ as shown in Figure 4. The heterodyne response of $1.6 \mathrm{~dB}$ at $4.2 \mathrm{~K}$ and $1.8 \mathrm{~dB}$ at about $2 \mathrm{~K}$ was measured with a $15-\mu \mathrm{m}$ beam splitter at the frequency of $935 \mathrm{GHz}$ (see Figure 5). The response increased to $2.1 \mathrm{~dB}$ with a thinner $(6 \mu \mathrm{m})$ beam splitter at about $2 \mathrm{~K}$ that yield $\mathrm{T}_{\mathrm{RX}}=260 \mathrm{~K}$ (DSB). The correction made for both beam splitters gave very close figures of about $245 \mathrm{~K}$ at $935 \mathrm{GHz}$. The water vapor absorption line at about $990 \mathrm{GHz}$ is clearly seen at the $\mathrm{T}_{\mathrm{RX}}$ data measured at $\mathrm{IF}=1.5 \mathrm{GHz}$ (see Figure 6).

The calculated reflection at the IF port vs. dynamic resistance of the mixer is presented in Figure 7. The complete capacitance of the structure, which includes the twin-SIS

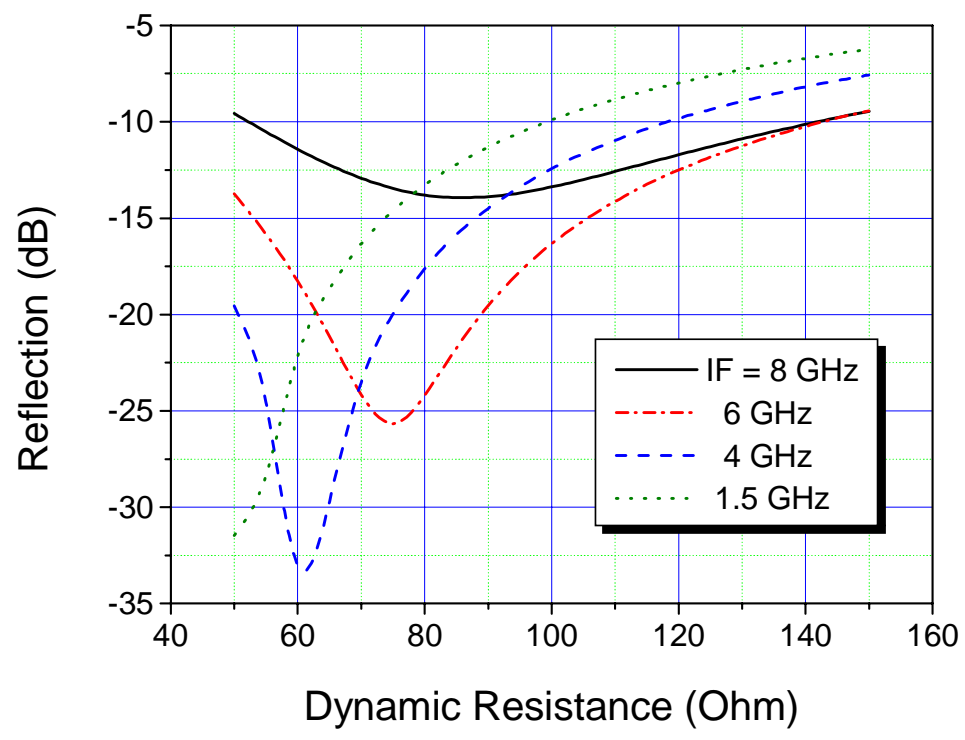

Fig. 7 Reflection loss at the IF port of the double-dipole antenna SIS mixer vs. dynamic resistance of the mixing element. Calculation made for four frequencies $1.5 \mathrm{GHz}, 4 \mathrm{GHz}, 6 \mathrm{GHz}$ and $8 \mathrm{GHz}$ in presence of inductive IF tuner (see Fig. 1).

junction, all microstrips and antenna filters, has been taken into account. The reflection below $-10 \mathrm{~dB}$ is predicted for most of the band $4-8 \mathrm{GHz}$. According to the calculations, such reasonable coupling to a $50 \Omega$ transmission line can be obtained with a series inductor, $\mathrm{L}=0.7 \mathrm{nH}$, which can be formed by two $1 \mathrm{~mm}$ long bond wires of $20-50 \mu \mathrm{m}$ in diameter separated at the distance of $0.2 \mathrm{~mm}$ (see Fig. 1).

The antenna beam is measured in the direct detection mode using a relatively narrow beam source that provide the dynamic range up to $40 \mathrm{~dB}$ (see Figure 8). The receiver was being rotated about the phase center of the antenna. It is found that the antenna mainlobe at $915 \mathrm{GHz}$ is almost round and its half-width is about $1.8^{\circ}$ at $-11 \mathrm{~dB}$, the first-order sidelobe appeared below $-16 \mathrm{~dB}$, and the beam quality is not degrading at $850 \mathrm{GHz}$. The width of the beam is somewhat decreasing at higher frequency that fit expectations for the diffraction limited optics. To obtain the exact beam width required by HIFI (F/3-F/5), an ellipsoid correction mirror is suggested in front of the mixer. The cross-polarized component of the beam is also measured at $850 \mathrm{GHz}$ and found to be below $-22 \mathrm{~dB}$. 


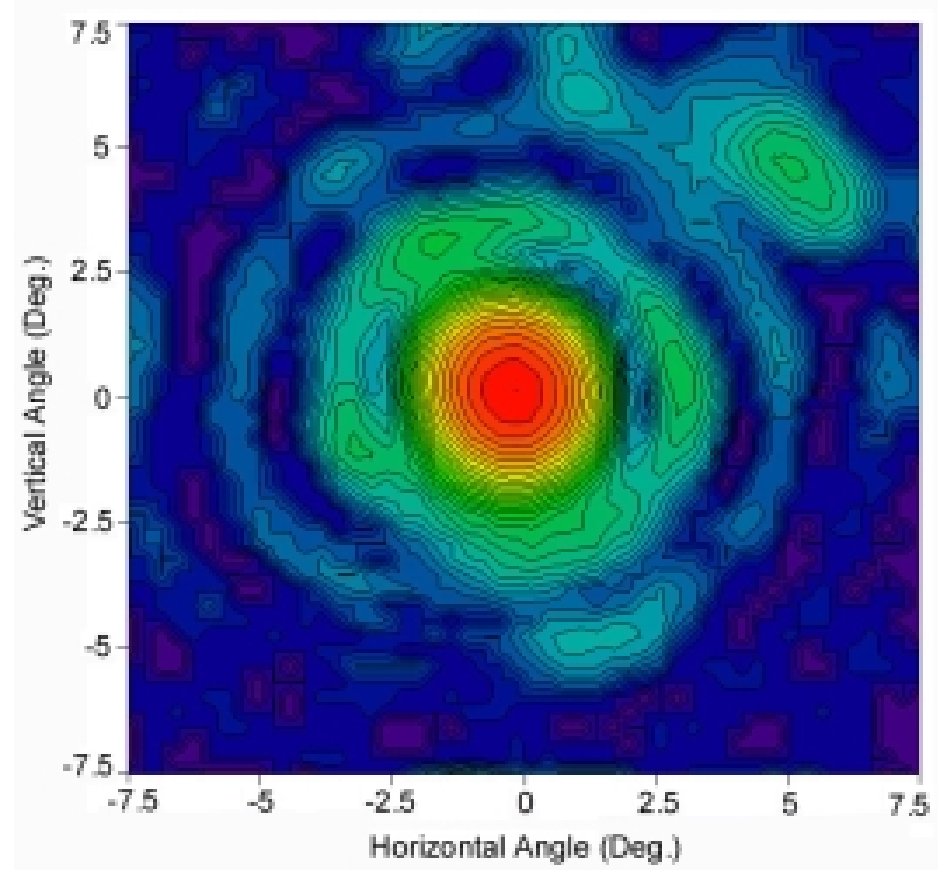

Fig. 8 Antenna beam pattern of experimental double-dipole SIS mixer with $\mathrm{NbTiN} / \mathrm{SiO}_{2} /$ Al tuning microstrip at $915 \mathrm{GHz}$. The chip is mounted at elliptical lens of diameter $10 \mathrm{~mm}$. Contours are at $1 \mathrm{~dB}$ step. All sidelobes are below $-16 \mathrm{~dB}$. The distant spot at the top-right corner is caused most probably by unknown reflection.

\section{Conclusions}

A double-dipole antenna THz-band SIS receiver with noise of only 10 photons is demonstrated experimentally. The receiver can cover HIFI band 3 and the part of band 4 demonstrating however cut-off just above $1 \mathrm{THz}$. We conclude that $\mathrm{NbTiN} / \mathrm{SiO}_{2} / \mathrm{Al}$ tuning microstrip sputtered at ambient temperature can be low loss at least up to $1 \mathrm{THz}$ yielding the SIS receiver noise temperature of $250 \mathrm{~K}$ at $935 \mathrm{GHz}$ and $360 \mathrm{~K}$ at $1 \mathrm{THz}$. These experimental results support the design value of effective sheet resistance of the $\mathrm{NbTiN} / \mathrm{SiO}_{2} / \mathrm{Al}$ microstrip in the range 0.1-0.15 $\Omega$. It is demonstrated numerically that IF coupling is achievable in the range of $4-8 \mathrm{GHz}$ for this type of SIS mixer. We may conclude that a diffraction limited double-dipole lens-antenna can provide effective $r f$ coupling for a THz-band SIS mixer with sidelobes less than $-16 \mathrm{~dB}$. The cross-polarized component is found below $-22 \mathrm{~dB}$. It looks worth to try the back reflector from $\mathrm{Al}$ or $\mathrm{NbTiN}$ that can further improve both the noise temperature of the receiver and the sidelobes of the array antenna.

\section{$\underline{\text { Acknowledgments }}$}

The authors thank Th. de Graauw and V.P. Koshelets for continuous encouraging and D. Nguyen for technical assistance. The work was supported in parts by the European Space Agency under ESTEC Contract No.11653/95, Russian SSP "Superconductivity", RFBR project 00-02-16270, INTAS project 97-1712 and ISTC project 1199.

\section{References:}

[1] HIFI for Herschel Space Observatory (known formerly as FIRST), http://www.sron.nl 
[2] J. R. Tucker, M. J. Feldman, "Quantum detection at millimeter wavelengths," Reviews of Modern Physics, vol. 57, No. 4, pp. 1055-1112 (1985).

[3] J. W. Kooi, M. Chan, B. Bumble, H. G. LeDuc, P. Schaffer, T. G. Phillips, "230 and $492 \mathrm{GHz}$ low noise SIS waveguide receivers employing tuned $\mathrm{Nb} / \mathrm{A} 10_{\mathrm{x}} / \mathrm{Nb}$ tunnel junctions," Int. J. IR and MM Waves, vol. 16, pp. 2049-2068 (1995).

[4] A. Karpov, J. Blondel, M. Voss, K. H. Gundlach, "A three photon noise SIS heterodyne receiver at submillimeter wavelength," IEEE Trans. Appl. Supercond., vol. 9, pp. 4456-4459 (1999).

[5] J. A. Stern, B. Bumble, H. G. LeDuc, J. W. Kooi, J. Zmuidzinas, "Fabrication and characterization of NbTiN based SIS mixers for use between 600 and $1200 \mathrm{GHz}$," Proc. 9 Int. Symp. Space Terahertz Technol., pp. 305-313 (1998).

[6] D. C. Mattis and J. Bardeen, "Theory of the anomalous skin effect in normal and superconducting metals," Phys. Rev., vol. 111, pp. 412-417 (1958).

[7] P. Dieleman, T. M. Klapwijk, J. R. Gao, H. van de Stadt, "Analysis of Nb superconductor-insulator-superconductor tunnel junctions with Al striplines for $\mathrm{THz}$ radiation detection," IEEE Trans. Appl. Supercond., vol. 7, pp. 2566-2569 (1997).

[8] H. van de Stadt, A. Baryshev, P. Dieleman, Th. de Graauw, T. M. Klapwijk, S. Kovtonyuk, G. de Lange, I. Lapitskaya, J. Mees, R. A. Panhuyzen, G. Prokopenko, H. Schaeffer, “A 1 THz Nb SIS heterodyne mixer with normal metal tuning structure,” Proc. 6 Int. Symp. Space Terahertz Technol., pp. 66-77 (1995).

[9] V. Yu. Belitsky, S. W. Jacobsson, L. V. Filippenko, S. A. Kovtonyuk, V. P. Koshelets, E. L. Kollberg, Proc. 4 Int. Symp. Space Terahertz. Technol., U. of California, Los Angeles, CA, p. 538 (1993).

[10] J. Zmuidzinas, H. G. LeDuc, J. A. Stern, and S. R. Cypher, "Two-junction tuning circuits for submillimeter SIS mixers," IEEE Trans. Microwave Theory Tech., vol. 42, No. 4, pp. 698-706 (1994). M. Bin, M. C. Gaidis, J. Zmuidzinas, T. G. Phillips, H. G. Leduc, "Low-noise $1 \mathrm{THz}$ niobium superconducting tunnel junction mixer with a normal metal tuning circuit," Appl. Phys. Lett., vol. 68, pp.1714-1716 (1996).

[11] V. Yu. Belitsky and E. L. Kollberg, "Superconductor-insulator-superconductor tunnel strip line: features and applications," J. Appl. Phys., vol. 80, pp. 4741-4748 (1996). Y. Uzawa, Z. Wang, A. Kawakami, and S. Miki, "Performance of all-NbN quasi-optical SIS mixers for the terahertz band", this conference.

[12] N. N. Iosad, V. V. Roddatis, S. N. Polyakov, A. V. Varlashkin, B. D. Jackson, P. N. Dmitriev, J.-R. Gao, T. M. Klapwijk, "Superconducting transition metal nitride films for THz SIS mixers”, Appl. Supercond. Conf. ASC'2000, Sept. 2000, Rep. 5MD04, to be published in IEEE Trans. on Appl. Supercond. (2001).

[13] D. F. Filipovic, S. S. Gearhart, and G. M. Rebeiz, "Double-slot antennas on extended hemispherical and elliptical silicon dielectric lenses," IEEE Trans. on Microwave Theory Tech., vol. 41, vol. 10, pp. 1738-1749 (1993). 
[14] M. J. M. van der Vorst, P. J. I. de Maagt and M. H. A. J. Herben, "Matching layers for integrated lens antennas," Proc. Int. Symp. Antennas, (JINA '96), pp. 511-515 (1996). M. J. M. van der Vorst, PILRAP, the software for design of integrated lensantennas.

[15] A. Skalare, Th. de Graauw, H. van de Stadt, "A planar dipole array antenna with an elliptical lens," Microw. and Opt. Technol. Lett., vol. 4., pp. 9-12 (1991).

[16] S. V. Shitov, V. P. Koshelets, A. B. Ermakov, L. V. Filippenko, A. M. Baryshev, W. Luinge, J.-R. Gao, "Superconducting chip receivers for imaging application," IEEE Trans. on Appl. Supercond., vol. 9, pp. 3773-3776 (1999).

[17] J. Zmuidzinas and H. G. LeDuc, "Quasi-optical slot antenna SIS mixers," IEEE Trans. Microw. Theory Tech., vol. 40, pp. 1797-1804 (1992).

[18] S. V. Shitov, A. M. Baryshev, V. P. Koshelets, J.-R. Gao, J. Jegers, W. Luinge, H. van de Stadt, Th. de Graauw, "Wide-band quasi-optical SIS mixers for integrated receivers up to $1200 \mathrm{GHz}$," Proc. 7 Int. Symp. Space Terahertz Technol., pp. 525-534 (1996).

[19] B. D. Jackson, N. N. Iosad, B. Leone, J. R. Gao, T. M. Klapwijk, W. M. Laauwen, G. De Lange, and H. Van de Stadt, "DC and RF Properties of Nb SIS Mixers with NbTiN Striplines," Proc. 10 Int. Symp. Space Terahertz Tech., p. 144 (1999). B. Leone, B. D. Jackson, J. R. Gao, and T. M. Klapwijk, "Geometric heat trapping in niobium superconductor-insulator-superconductor mixers due to niobium titanium nitride leads," Appl. Phys. Lett., vol. 76, pp. 780-782 (2000).

[20] B. Jackson, G. de Lange, W. M. Laauwen, J. R. Gao, N. N. Iosad, T. M. Klapwijk, "NbTiN/SiO2/NbTiN and $\mathrm{NbTiN} / \mathrm{SiO} 2 / \mathrm{Al}$ tuning circuits for $1 \mathrm{THz}$ waveguide SIS mixers," Proc. 11 Int. Symp. Space Terahertz Technol., pp. 238-250, U. of Michigan, Ann Arbor, Michigan (1-3 May 2000).

[21] M. M. Khapaev, "Extraction of inductances of a multi-superconductor transmission line”, Supercond. Sci. Technol., vol. 9, pp. $729-733$ (1996). M. Yu. Kupriyanov, Private communication on the inductance calculation.

[22] R. L. Kautz, "Miniaturization of normal-state and superconducting striplines," $J$. Res. Natl. Bur. Std., vol. 84, pp. 247-259 (1979).

[23] A. M. Baryshev, B. D. Jackson, G. de Lange, S. V. Shitov, N. Iosad, J. R. Gao, T. M. Klapwijk, "Quasi-optical terahertz SIS mixer," Proc. 11 Int. Symp. Space Terahertz Technol., pp. 129-138, U. of Michigan, Ann Arbor, Michigan (1-3 May, 2000). B. D. Jackson, A. M. Baryshev, G. de Lange, W. M. Laauwen, L. de Jong, J. R. Gao, N. N. Iosad, T. M. Klapwijk, S. V. Shitov, "The Development of THz SIS mixers for HIFI," this conference. 